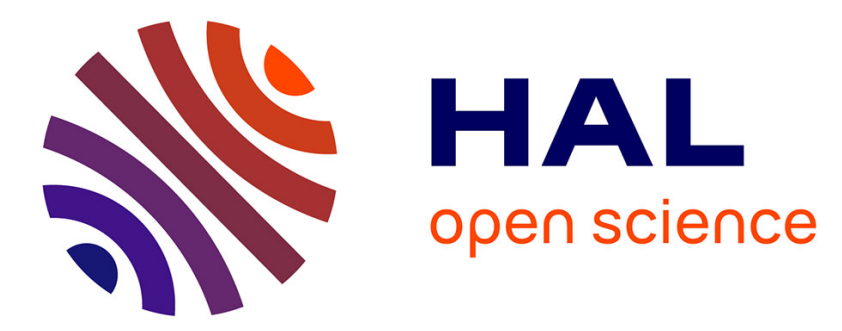

\title{
Shared Interaction on a Wall-Sized Display in a Data Manipulation Task
}

\author{
Can Liu, Olivier Chapuis, Michel Beaudouin-Lafon, Eric Lecolinet
}

\section{To cite this version:}

Can Liu, Olivier Chapuis, Michel Beaudouin-Lafon, Eric Lecolinet. Shared Interaction on a WallSized Display in a Data Manipulation Task. Proceedings of the 34th international conference on Human factors in computing systems, SigCHI; ACM, May 2016, San Jose, United States. pp.1-12, 10.1145/2858036.2858039 . hal-01275535

\section{HAL Id: hal-01275535 \\ https://hal.inria.fr/hal-01275535}

Submitted on 17 Feb 2016

HAL is a multi-disciplinary open access archive for the deposit and dissemination of scientific research documents, whether they are published or not. The documents may come from teaching and research institutions in France or abroad, or from public or private research centers.
L'archive ouverte pluridisciplinaire HAL, est destinée au dépôt et à la diffusion de documents scientifiques de niveau recherche, publiés ou non, émanant des établissements d'enseignement et de recherche français ou étrangers, des laboratoires publics ou privés. 


\title{
Shared Interaction on a Wall-Sized Display in a Data Manipulation Task
}

\author{
Can Liu $^{1,2,3}$ Olivier Chapuis ${ }^{1,2}$ Michel Beaudouin-Lafon ${ }^{1,2}$ Eric Lecolinet $^{3}$ \\ ${ }^{1}$ Univ Paris-Sud \& CNRS (LRI) \\ ${ }^{2}$ INRIA \\ Université Paris-Saclay \\ F-91405 Orsay, France \\ Université Paris-Saclay \\ F-91405 Orsay, France
${ }^{3}$ Telecom ParisTech \& CNRS (LTCI)
Université Paris-Saclay
F-75013 Paris, France

\begin{abstract}
Wall-sized displays support small groups of users working together on large amounts of data. Observational studies of such settings have shown that users adopt a range of collaboration styles, from loosely to closely coupled. Shared interaction techniques, in which multiple users perform a command collaboratively, have also been introduced to support co-located collaborative work. In this paper, we operationalize five collaborative situations with increasing levels of coupling, and test the effects of providing shared interaction support for a data manipulation task in each situation. The results show the benefits of shared interaction for close collaboration: it encourages collaborative manipulation, it is more efficient and preferred by users, and it reduces physical navigation and fatigue. We also identify the time costs caused by disruption and communication in loose collaboration and analyze the trade-offs between parallelization and close collaboration. These findings inform the design of shared interaction techniques to support collaboration on wall-sized displays.
\end{abstract}

\section{Author Keywords}

Co-located collaboration; shared interaction; collaboration

styles; wall-sized display; classification task; pick-and-drop

\section{ACM Classification Keywords}

H.5.2 [Information Interfaces and Presentation]: User Interfaces - Graphical user interfaces

\section{INTRODUCTION}

Wall-sized displays are well-suited to small groups of users working together on large amounts of data: Multiple users can move freely in front of the display to get an overview or see details without changing its content [2], leading to a variety of collaboration styles [11]. Previous work has demonstrated the benefits of such displays in various tasks including sensemaking [1], way-finding [17], searching [22] and exploratory visual analysis [21]. Liu et al. [13] showed the performance advantage, as the amount of data increases, of using a ultra-high resolution wall-sized display vs. a classical desktop setup for a single-user classification task. This task

\footnotetext{
Can Liu, Olivier Chapuis, Michel Beaudouin-Lafon, Eric Lecolinet. In CHI '16: Proceedings of the 34th international conference on Human factors in computing systems, 12 pages, ACM, May 2016. to appear.

(C) ACM, 2016. This is the author's version of the work. It is posted here by permission of ACM for your personal use. Not for redistribution. The definitive version will be published in CHI'16, May 07-12, 2016, San Jose, CA, USA http://dx.doi.org/10.1145/2858036.2858039
}

required users to manipulate data, not just observe or navigate it. Indeed, in many cognitive tasks such as sense-making [3] or problem-solving [11], users need to reorganize data items to solve the task. However, little research has studied the effects of shared interaction on co-located collaborative work that involves data manipulation in such settings.

Designing effective interaction for collaboration requires a deep understanding of situations involving complex phenomena when multiple users interact with the artifacts as well as communicate with each other [28]. Previous studies of group work on large displays have consisted mostly of observational studies identifying various collaborative strategies, from loosely to tightly coupled work, when performing a task such as drawing [26], writing [4], route finding [25], visual analytics [10], problem solving [11] or sense-making [27]. While observational studies capture high-level phenomena, we believe that they should be complemented by controlled laboratory studies to better understand low-level phenomena occuring at the articulatory level in collaborative work. Such knowledge can inform the design of more effective techniques to support collaboration.

Collaborative interaction in large physical spaces can be made more efficient by designing shared interaction techniques where multiple users each perform a part of a command or basic task $[15,20]$. This may improve both performance and user experience by supporting new forms of division of labor. However, the application of such techniques to wall-sized displays has received little attention and we are not aware of any formal study quantifying the effect of shared interaction on collaboration and productivity.

In order to start filling this gap, we conducted an experiment to study the effects of a shared interaction technique on the articulatory aspects of a collaborative data manipulation task on a wall-sized display. We used an abstract task from previous work [13] to focus on the interaction level of a collaborative task and to enable in-depth quantitative analyses. Pairs of participants performed a classification task by picking and dropping data items scattered over the large surface. We compared five collaboration styles that were operationalized by crossing two dimensions - task parallelization and shared interaction support. The findings deepen our understanding of collaborative interaction and can inspire the design of new techniques supporting collaboration.

The rest of this paper reviews related work, motivates our approach with real-world tasks and describes how we have operationalized the collaborative styles to cover a range of 
coupling types between users and assess the effects of shared interaction support. We then describe the experiment, present the findings drawn from the analysis of the data, and discuss the possible implications of these findings. We conclude with a summary of contributions and directions for future work.

\section{RELATED WORK}

\section{Collaborative Behaviors with Large Displays}

Collaborative behaviors when using large displays have been studied mostly in the context of tabletop environments. Scott et al. [23] found that people use three types of territories (personal, group and storage) to help coordinate their interactions during tabletop collaboration. Tang et al. [25] observed pairs of users working around a tabletop and identified six typical collaborative coupling styles: same problem, same area; one working, another viewing in an engaged manner; same problem, different areas; one working, another viewing; one working, another disengaged; and different problems. Isenberg et al. [10] studied a system supporting collaborative visual analytics tasks on a tabletop and identified eight types of collaborative styles ranging from loose to close collaboration.

Although these results may be applicable, at least partially, to wall-sized displays, there are some major differences between vertical and horizontal settings. User mobility is higher when working with vertical displays, especially ultra-high resolution large wall-sized displays, which promote physical navigation for viewing large amounts of data [2].

Jakobsen \& Hornbaek [11] observed pairs performing a collaborative sense-making task on a multitouch wall-sized display. Their findings about coupling styles and users' proximity show that simultaneous input reduces the need for coordination for loosely coupled work. Moreover, collaborating groups did not divide the display into physical territories, but instead divided the tasks and switched fluidly between parallel and joint work.

While observational studies provide rich insights about how people collaborate, little research has so far assessed the costs and benefits associated with different collaboration styles, and even fewer studies have attempted to quantitatively assess the effect of interaction. A rare example is Hawkey et al. [7], who conducted an experiment that varies the proximity among participants and with the shared display and showed that participants preferred to work closely together.

Our work builds on these qualitative findings and goes one step further by operationalizing different collaboration styles so that their effects can be measured quantitatively. The task we propose is flexible enough to support different levels of coupling - from loose to close collaboration - and to understand their respective characteristics and behavior patterns.

\section{Shared Interaction Techniques}

While basic input techniques have been studied in collaborative settings, e.g., $[9,16]$, only a few interaction techniques have been designed to support tightly coupled collaboration in large interactive spaces. For example, Cooperative Gestures [15] let multiple users issue part of a gesture to collectively specify a command in a tabletop application for photo manipulation. Synchronous gestures [20] explore the simultaneous use of multiple devices to enter commands, such as bumping them together or performing synchronous stitching gestures. By allowing users to share knowledge and actions across space, such techniques could be helpful for close collaboration over large surfaces.

We are not aware, however, of formative studies evaluating the effects of shared interaction techniques on co-located collaborative work. The study reported in this paper compares pairs manipulating data with and without a shared interaction technique in different collaborative styles. Our goal is to gain a deeper understanding of the costs and benefits of shared interaction in the different styles and to inspire the design of new interaction techniques to support collaboration.

\section{Quantitative Methods for Studies of Collaboration}

Ethnographic approaches capture rich phenomena from the real world, but are not designed to control experimental variables. Controlled experiments can therefore complement ethnographic studies, and be informed by them, in order to systematically compare different conditions and help detect or confirm causal effects.

Several methodologies have been proposed to study collaboration in a formative way. Pinelle et al. [19] describe a task modeling scheme to facilitate the evaluation of groupware. Quantitative measures are collected by counting the actions according to the articulatory aspects of the coordination. Other methods attempt to measure the equity of contribution from group members, for instance by counting the interactions made by individuals [14] or by considering the Gini Coefficient [5]. Tan et al. [24] designed a job-scheduling task for evaluating coordination in group work. This task enforces collaboration and coordination and makes it possible to measure performance against an optimal solution.

The experiment reported in this paper follows this line of work. We use an abstract task inspired by observations of real-world tasks and we operationalize collaborative situations with several levels of coupling. We quantify the effectiveness of communication and the amount of collaboration, and illustrate behavior patterns with objective measures.

\section{MOTIVATION}

Liu et al. [13] illustrate the need for users to manipulate data on a wall-sized display in some real-world tasks: Program committee chairs used a wall-sized display to assign papers to associate chairs (ACs); They moved undecided papers from a shared area into columns of papers, one per AC, trying to keep their workload balanced. Neuroanatomists used a wall-sized display to compare and classify a set of 3D brain scans; They moved the images to compare them more easily. Conference chairs used a wall-sized display to schedule a large conference with many parallel tracks; Up to twelve people worked in pairs or triads to move talks into proper sessions based on complex constraints. The authors observed a range of collaboration styles, from loose to close collaboration, such as collaborators working independently, or asking each other's opinion about a proper session for a talk, or working together to find such a session. 
This is consistent with our experience of observing and interviewing users working with large datasets. We interviewed social scientists who performed a collaborative task with a large number of graphs displayed on a large touch screen $(4 \mathrm{~m} \times 1.15 \mathrm{~m})$. Each graph visualized a person's social network and they attempted to find correlations between social behavior and eating disorder. They put all the graphs on the display and invited professionals with different expertise, including statisticians and ethnographers, to look at them together. Their goal was to combine the experts' knowledge, to enable discussions, to define a common vocabulary based on the topology and arrangement of the graphs, and to spark ideas about how to extract common features.

Three experts conducted a collaborative session on the display, using basic touch input for dragging and rotating the graph images. They moved the graphs around, either to try out groupings while discussing, or to tell a story by laying out several graphs. In the interview, one sociologist mentioned physical fatigue as a problem, due to the well-known gorilla-arm effect [8]. Collaborators also often passed content around to reduce the amount of walking.

Similar to the conference scheduling task observed by Liu et al. [13], this task also includes complex sense-making and resource allocation. The common element is that users need to move scattered data elements in order to progressively understand, find correlations, or organize them. We believe that one of the major advantages of using a wall-sized display is to encode meaning in the spatial relationships [1]. The display provides a common reference for deictic actions among users and enables group dynamics based on shared knowledge and mutual awareness.

In this paper, we use a similar approach to Liu et al. [13] in order to focus on understanding some fundamental phenomena at the interaction level when multiple users manipulate a large data set in a collaborative task.

\section{Abstract Classification Task}

The classification task from Liu et al. [13] abstracts a realworld data manipulation task. The task consists of moving misclassified discs into the appropriate container according to their label. Misclassified discs are colored in red, and turn green when moved into a container with a majority of discs with the same label. Participants move discs using a pointing device to control a cursor and a single button to pick and drop the discs with a click. The task is completed when the discs in each container have the same label, i.e. when all discs are green (Figure 1). The task was used to compare the performance of single users when using a desktop vs. a wall-sized display. It was designed to eliminate as much as possible the domain-dependent mental work involved in a real-world task, so that the difference in performance could be attributed to the main factors in the experiment.

Jakobsen and Hornbæk [12] used this task to test the effects of locomotion on interaction with a wall-sized display. Building on this line of work, we also use this task, with different factors, to study interaction phenomena when pairs of users perform a collaborative task that involves data manipulation.

\begin{tabular}{|c|c|c|}
\hline $\begin{array}{l}\text { Collab. Interaction } \\
\text { Coupling }\end{array}$ & Not provided & Provided \\
\hline Loose & LooseComm & LooseTech \\
\hline Close & CloseComm & CloseTech \\
\hline
\end{tabular}

Table 1. Four collaboration styles generated by crossing two dimensions. Collaborative coupling is loose when co-workers work independently, and close when they work together. Shared interaction is enabled by the drop-for-partner technique, which lets a partner drop the disc picked by the other partner. When shared interaction is not provided, partners can help each other only by communicating with each other.

\section{OPERATIONALIZATION}

In real-world tasks, users engage in different styles of collaboration and switch fluidly among them $[10,11,25]$ : collaborators can work independently of each other or, at the other extreme, work closely together. In the context of a controlled experiment where we want to study the effects of different collaboration styles on interaction, we need to operationalize these collaboration styles and control which style the participants can use in each condition.

Liu et al.'s abstract classification task [13] is a good candidate for operationalizing collaboration styles because the task can be executed either with or without co-workers explicitly helping each other. It is also possible to vary the difficulty of the task by controlling the initial layout of the discs.

\section{Collaboration Styles}

We operationalize the collaboration styles by crossing two dimensions (Table 1). The first dimension, collaborative coupling, is operationalized by task parallelization: Loose collaboration is operationalized by letting the co-workers perform the subtasks (moving a disk) independently; Close collaboration is operationalized by forcing them to perform each subtask together. The other dimension is the availability of shared interaction support. We use a simple technique designed for this task and observe how it affects collaboration, efficiency and subjective experience.

\section{Task Parallelization: Loose vs. Close Collaboration}

The overall task of classifying the red discs is performed by a series of individual pick-and-drops. These subtasks can be performed by a pair of users either in parallel or sequentially, representing two basic patterns for the division of labor. In the parallel case, users work independently of each other: each user picks one of the remaining red discs and puts it in an appropriate container. In the sequential case, the pair performs each pick-and-drop in tight collaboration. This style is enforced by allowing only one disc to be picked at any one time. While the parallel case results in users solving the sub-tasks concurrently, the sequential case can benefit from partners helping each other.

\section{Shared Interaction Support}

Beyond the level of coupling, collaboration in the present task is also affected by how users help each other. Based on our observation of sociologists in their collaborative task, where they discussed about data items and passed them among collaborators, we designed a simple technique to support collaborative interaction. We call it drop-for-partner. 


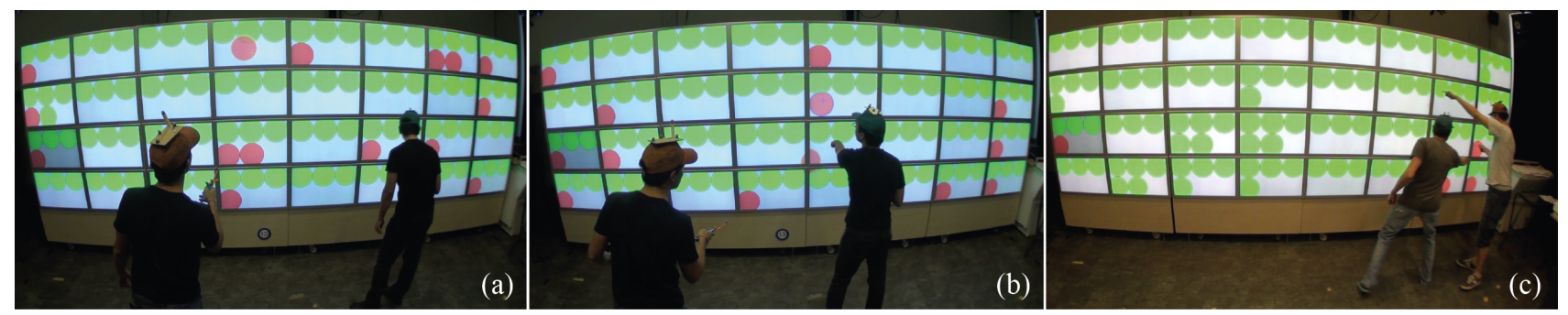

Figure 1. Pair behaviors with different collaboration styles. (a) Participants working on separate discs. (b) One participant shows his partner the target container while moving his own disc. (c) Under Close Comm condition, the participant on the right is telling his partner "move this one there".

Each user can spell out the label of the disc they have picked so that the other user may indicate a destination container if they see or remember one. With drop-for-partner, instead of indicating the destination container, the other user can finish the pick-and-drop action on behalf of the first user by dropping her disc, e.g. with a dedicated button on his device. In other words, a user can drop the disc picked by her partner at the location of the user's cursor.

Drop-for-partner is a simple shared interaction technique that requires minimal technical support and that can be used in a variety of tasks. The purpose is to compare collaboration with shared interaction support vs. without it, i.e. with verbal or gestural communication only. Both cases require the users to coordinate and synchronize their actions, so the benefit of saving time by having the other user look for a container or move the disc to its destination may be offset by the coordination overhead.

Crossing the above two dimensions, Loose vs. Close Collaboration and Communication Only vs. Shared Interaction, leads to four collaboration styles. We add a baseline condition, Divide \& Conquer, to contrast with the other four explicit collaborative styles:

- Divide\&Conquer: the task is performed in parallel and the partners must not communicate nor help each other. No spatial division is enforced, participants can move freely.

- LooseComm: as above, but the partners are allowed to communicate. They receive instructions encouraging them to help each other by showing their partner a correct container when they can (Figure 1-b).

- LooseTech: as above, but the partners can also help each other by using the drop-for-partner technique.

- CloseComm: the task is performed sequentially. Each pair has a single input device so that only one partner can perform the pick-and-drops. The other partner is encouraged to help, e.g. by searching for containers or items.

- CloseTech: as above, but the second partner also has an input device, which can only be used to drop the disc picked by the first partner (drop-for-partner).

As discussed before, these conditions will let us compare interaction phenomena across three collaborative coupling levels: no coupling (baseline), loose coupling and close coupling. They will also let us compare the effects of shared interaction on loose vs. close collaboration. Note that while the task involves communication and synchronization between users, interruptions, and the use of short-term memory, these are tightly bound to the articulatory level of collaboration. By choosing an abstract class where the decision-making process is minimal, we limit the higher-level cognitive processes such as discussing or arguing about a choice. Yet, at the interaction level, the task is representative of a large class of real-world situations such as those outlined in the previous section.

\section{Layout Locality}

Liu et al. [13] controlled information density by the size of the textual labels. We use the smallest size (12 pt) corresponding to the higher information density. Participants must be close to the display to read the labels and must move around to read the labels of discs that are further away than the adjacent columns (Figure 1). Liu et al. [13] operationalized the difficulty of the task by using different numbers of categories ( 2 for Easy tasks and 4 for Hard tasks). We use 8 categories which, combined with the small label size, results in a task hard enough that collaboration is likely to be beneficial.

In order to operationalize the distribution of information on the wall, we use two types of layouts, Local and Distant, by controlling the distance of misclassified discs to the closest appropriate container. With Local layouts, all pick-and-drops can be done between adjacent containers: the distance between a pick and a drop is short enough that users only need to search the area in front of them. With Distant layouts, all pick-and-drops involve containers in non-adjacent columns: users must move in order to search a larger part of the display to find a proper container.

These two types of layouts are likely to encourage or favor different collaboration styles. Distant layouts simulate realworld situations where a large amount of data is scattered over the whole space, forcing users to manipulate data across large distances and favoring tighter coupling so that, e.g., a user can drop the partner's disc at a distance. Local layouts simulate the opposite situation where work can be done locally.

\section{EXPERIMENT}

The goal of this experiment is to understand how collaborative interaction affects efficiency and, more precisely, the effects of using a shared interaction technique in different collaboration styles. We compare five collaboration styles (STYLE = Divide \&Conquer, LooseComm, CloseComm, Clos eTech, LooseTech) for two types of layouts (LAYOUT = Local, Distant) and analyze the amount of collaboration and coordination, performance at the overall task level and individual pick-and-drop level, patterns of physical navigation and subjective ratings. 
The experiment addresses the following research questions:

- What are the efficiency costs and benefits, at the interaction level, when pairs of users manipulate data on a wall-sized display in loose vs. close collaboration settings?

- What are the effects of providing a shared interaction technique on collaboration behavior, efficiency, physical navigation and subjective assessment?

- Do the effects depend on the spatial distribution of data?

\section{Participants}

We recruited ten pairs of volunteers, age 21 to 40, all with normal or corrected-to-normal vision. The participants were students and engineers from the local university. Five pairs were male-only, one female-only, four mixed. The participants of seven of the pairs knew each other as colleagues or fellow students.

\section{Apparatus}

We use a $5.5 \mathrm{~m} \times 1.8 \mathrm{~m}$ wall-sized display consisting of an $8 \times 4$ matrix of 30 " LCD panels with overall resolution $20480 \times 6400$ pixels (Figure 1), driven by a cluster of 16 Apple Mac Pro computers running Linux and controlled by a front-end computer via a dedicated network. The software is implemented using the jBricks Java toolkit [18].

The input device is a motion-tracked pointer, controlling a cursor on the wall using raycasting, mounted on a mobile phone. The orientations and positions of the participants' heads and their pointing devices are tracked by a VICON motion-capture system. The mobile phones are running an Android application that communicates with the front-end computer via OSC messages. The interface features one or two buttons according to the condition. One button is used for picking and dropping, the other button for the drop-forpartner feature.

We use raycasting instead of a mobile trackpad as in [13] because raycasting provides more awareness of the other user's actions (an important point in collaborative interaction [6]) and leaves one hand free to perform communicative gestures. We did not use direct touch input because some areas of the wall are hard to reach with the hands. Moreover, direct touch requires close proximity with the display, while we expect users to be able to pick and drop at a distance, e.g. when the other user is showing the target container.

\section{Task Details}

Figure 1 shows participants running the experiment. Each screen of the display serves as a container and can hold a maximum of 6 discs. Each task begins with a layout containing 16 misclassified discs colored in red, all the other discs being already classified and colored in green. Once a disc is moved to a correct container, it becomes green. The participants need to perform at least 16 pick-and-drop operations to complete the task. The 8 label categories are $\mathrm{C}, \mathrm{D}, \mathrm{H}, \mathrm{N}, \mathrm{K}$, $\mathrm{R}, \mathrm{X}, \mathrm{Z}$.

A layout satisfying the constraints of each LAYOUT condition is generated randomly and ten additional layouts are derived from it by performing horizontal, vertical and central symmetries and by permuting the labels [13]. This ensures that the tasks are of equal difficulty while minimizing the learning effect resulting from the memorization of the layouts.

\section{Procedure}

The experiment is a $[5 \times 2]$ within-participants design with factors STYLE and LAYOUT. The participants begin by reading a standard explanation of the task and perform an initial four-trial training session, two for each LAYOUT. They are told to get familiar with the task and can practice the dropfor-partner technique. During the training, freeform collaboration is encouraged by the experimenter, who also recommends participants to find an efficient and comfortable way to communicate and coordinate by establishing oral protocols (except, of course, for Divide \&Conquer). The participants are given the following examples, which emerged spontaneously during pilot studies: 1) Say "I have [letter]" after picking a disc; 2) Say "I drop [letter]" when dropping a disc; 3) Say "I drop [letter] for you" before dropping a disc for the partner. Note that participants are not required to collaborate or to use any protocol. The instructions during training are reminders that they should optimize their coordination.

After training, the participants are instructed to perform the task under the rules of each collaboration style. To study the effect of locality, participants are told about the differences between Local and Distant layouts. For all conditions, participants are asked to complete the task as quickly as possible but to avoid dropping discs into the wrong containers.

The experiment is blocked by STYLE, then by LAYOUT. There is one training trial and two replications for each STYLE $\times$ LAYOUT condition. We use the same 20 layouts (5 STYLE $\times 2$ LAYOUT $\times 2$ replications) for each pair, distributed so that each layout is used with each STYLE condition the same number of times. The order of STYLE blocks is counterbalanced across pairs using a Latin Square and the order of LAYOUT is swapped for each strategy to counterbalance this order with STYLE. In the CloseComm condition each participant is given the input device for one trial, then the roles are exchanged in the second replication (a similar policy is used with CloseTech). The experiment lasts about 70 minutes.

\section{Data collection}

We collected 200 measured trials $(5$ STYLE $\times 2$ LAYOUT $\times$ 2 replications x 10 groups). We measure Task Completion Time, $T C T$, and count misplaced discs as errors. We recorded the experiment on video and logged kinematic data about the interactions and the participants' physical navigation using the motion tracking system.

During the experiment, two experimenters, one monitoring each participant, use a mobile device to measure the effectiveness of the collaboration. The mobile device features three buttons that record the following actions in the kinematic log: (1) "Asked for Help" when the participant tells the letter on his disc to his partner, e.g. "I have D"; (2) "Effective Help" when the participant finds a correct container for his partner and the disc is dropped into this container; (3) "Pick Conflict" when the partners try to pick the same circle.

At the end of the experiment, the participants fill out a questionnaire about their assessment of each collaboration style. 


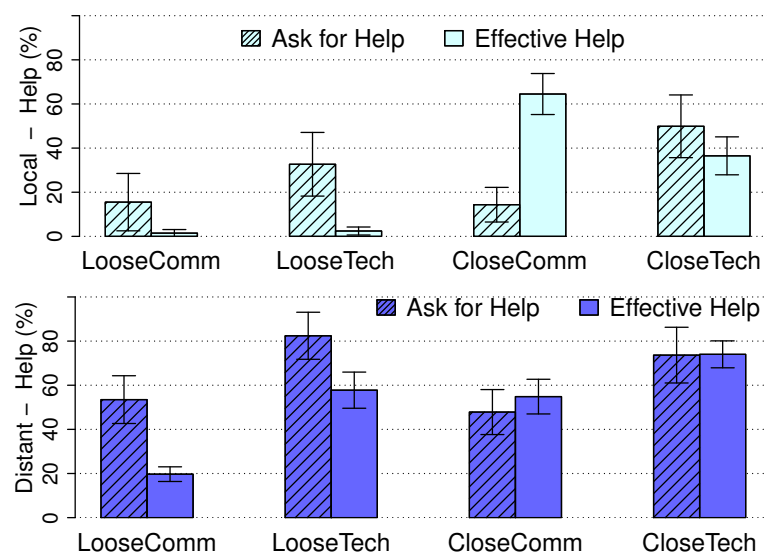

Figure 2. Percentage of pick-and-drop by STYLE and LAYOUT (top: Local, bottom: Distant) where a participant asked for help (hashed) and effectively helped their partner (solid).

\section{RESULTS}

Our analyses ${ }^{1}$ are focused primarily on the the effects of the drop-for-partner shared interaction technique on the different collaborative styles, for the two types of layout. We first look at the number of errors and conflicts, and then quantify the effective collaboration between participants in terms of pickand-drop actions that involved help. We then analyze time performance for the overall task and for individual pick-anddrop actions to better understand the costs and benefits of each condition, and look at physical navigation patterns to get a deeper understanding of the collaboration patterns. Finally we analyze the subjective assessment of the task by the participants.

\section{Errors \& Conflicts}

On average, participants made 0.88 erroneous pick-anddrop per trial, from 0.5 for CloseTech-Distant to 1.45 for LooseComm-Distant, but the differences are not significant ( $p=0.12, p$ 's $>0.3$ for all the other comparisons). Moreover, we logged only 6 pick conflicts overall, 5 of which occurred with different groups, but in the same condition: Divide\&Conquer-Distant. This suggests that pairs are generally aware of the partner's position or action and avoid conflicts, although performing the task independently may lead to slightly more conflicts. It also means that differences between conditions cannot be attributed to errors and conflicts.

\section{Effective Collaboration}

Figure 2 shows the proportion of pick-and-drops that involve explicit help by the other partner for each collaborative condition $^{2}$. (Divide\&Conquer is excluded because explicit collaboration is forbidden in this condition.) For each trial we count the number of times participants asked for help (by spelling out the letter on the disc they picked) as well as the number of pick-and-drops completed with effective help from the partner. This provides a quantitative measure of the amount of collaboration. Since communication with the partner may

\footnotetext{
${ }^{1}$ We briefly report on ANOVAs and use paired (by pairs) t-tests (and Wilcoxon rank sum tests for errors, conflicts and the subjective data), both with Bonferroni correction.

${ }^{2}$ In all barplots, error bars show the $95 \%$ confidence interval.
}

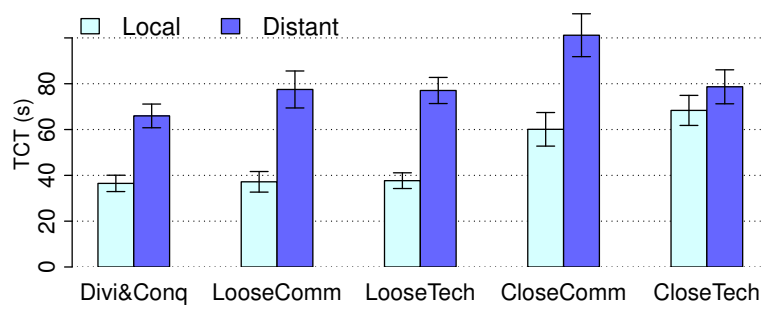

Figure 3. Mean TCT by STYLE $\times$ LAYOUT conditions.

provide beneficial help but also takes time, this also reflects part of the efficiency trade-off of collaboration.

For loose collaboration, we observe that for Local layouts, participants did not help each other, and, indeed, the experimenters noted that the participants performed the task similarly to Divide\&Conquer, except for spelling out the disc they picked from time to time. By contrast, for Distant layouts with loose collaboration, participants helped each other significantly more often for LooseTech than for LooseComm $(60 \%$ vs. $20 \%, p<0.001)$. Thus, the shared interaction technique was used heavily in LooseTech even though the task was performed in loose collaboration.

For close collaboration, participants helped each other in both layout conditions. For the Distant layouts with drop-forpartner, about $75 \%$ of the pick-and-drops were performed in collaboration, showing indeed a very tight collaboration. Without interaction support, this number dropped (significantly, $p<0.001$ ) to $57 \%$. With the Local layouts in close collaboration, the situation is reversed: we have significantly less effective help with the shared interaction technique that without (35\% for CloseTech vs. 63\% for CloseComm, $p<$ 0.001). Moreover, for CloseComm, the help is provided spontaneously, with few instances of "Ask for Help".

In fact, the experimenters observed that the participant without the pointer was very close to her partner and often became very proactive by planning ahead the next pick-and-drops for her partner (e.g., "put this H there"), see Figure 1-c. This created an effective division of labor where one partner focused on manipulating and the other on planning. By contrast, with drop-for-partner, the participant who was not allowed to pick waited for her partner to tell the label. It is worth noting that although participants were free to use verbal and gestural communication or drop-for-partner to collaborate, almost all instances of help in Distant layouts are through drop-forpartner when it is available.

Overall, these results demonstrate that both the spatial distribution of information (Distant vs. Local) and the availability of shared interaction support (drop-for-partner) had a large influence on collaborative behaviors at the interaction level.

\section{Performance Costs and Benefits}

Task Completion Time

We first look at the Task Completion Time (TCT) for each condition shown in Figure 3. An ANOVA in the model $T C T \sim$ STYLE $\times$ LAYOUT $\times$ Rand(PARTGROUP) shows significant effects of STYLE $\left(F_{4,36}=33.0, p<0.001, \eta_{G}^{2}=0.46\right)$ and $\operatorname{LAYOUT}\left(F_{1,9}=244, p<0.001, \eta_{G}^{2}=0.64\right)$, and a sig- 
nificant STYLE $\times$ LAYOUT interaction $\left(F_{4,36}=13.2, p<0.001\right.$, $\left.\eta_{G}^{2}=0.19\right)$. As expected, the pairs are significantly faster with Local layouts than with Distant ones $(48.0 \pm 7.1 \mathrm{~s}$ vs. $80.1 \pm 7.8$ s). Comparing Local and Distant layouts by STYLE shows that Local and Distant are significantly different for each STYLE ( $p$ 's $<0.001)$, except for CloseTech $(p=0.0983)$.

For Local layouts, we first note that TCT for Divide\&Conquer, Loose Comm and LooseTech (the parallel styles) are very close and not significantly different $(p$ 's $=1.0)$. This is expected, since in these cases the pairs perform the task in a similar way, using a divide \& conquer strategy. Moreover, not surprisingly, all these styles are significantly faster than the sequential styles ( $p$ 's $<0.001)$, and there is no significant difference between CloseComm and CloseTech $(p=0.15)$. Thus, in particular, drop-for-partner does not improve efficiency when data manipulation is local.

For Distant layouts, Divide\&Conquer is marginally faster than LooseTech $(p=0.072)$ and significantly faster than all the other styles $(p$ 's $<0.031)$. This result highlights the cost of explicit collaboration. Moreover, LooseComm, LooseTech and CloseTech are very close in performance (the differences are not significant, $p$ 's $=1.0$ ) for Distant layouts. Note that with drop-for-partner, close collaboration (sequential pick-anddrops) has similar performance to loose collaboration (parallel pick-and-drops). This style is also significantly faster than CloseComm $(p<0.001)$, showing that drop-for-partner could improve task performance for Distant layouts ${ }^{3}$.

While we have noticed different collaborative behaviors across the different styles (see the Effective Collaboration sub-section above), in particular for Distant layouts, overall performance is very similar for some of them. To better understand the underlying trade-offs, the rest of this section analyzes interaction at the level of individual pick-and-drops. In particular, the goal is to understand the good performance of CloseTech relative to the parallel styles, and why LooseTech does not outperform the other parallel styles.

\section{Individual Pick-and-Drops}

We analyze the movement time $\left(M T_{P D}\right)$ of 3200 pick-anddrops (160 per condition after removing the erroneous ones). Figure 4 shows the average $M T_{P D}$ per STYLE $\times$ LAYOUT.

Again, for Local layouts, there is no difference between $D i$ vide\&Conquer, LooseComm and LooseTech (same behavior of the pairs). Indeed, there is no significant difference among the STYLE conditions with Local layouts.

For Distant layouts, both close collaboration styles are significantly faster than the loose collaboration styles $(p$ 's $<0.006)$. This shows that close collaboration increases the efficiency of individual pick-and-drops. In particular, individual pickand-drops are twice as fast with CloseTech than with $\mathrm{Di}$ vide \&Conquer. But this advantage is offset by the fact that the 16 pick-and-drops have to be performed sequentially vs.

\footnotetext{
${ }^{3}$ Although CloseComm is slower than the other styles for Distant layouts $(p$ 's $<0.001)$, it is 1.3 times faster than twice the TCT of Divide \&Conquer which, according to our pilot studies, is a close approximation of the time it would take a single user to perform the task. Thus, help from the partner did improve performance.
}

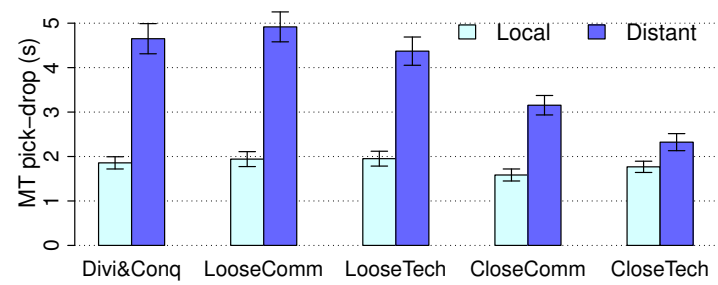

Figure 4. Mean time to perform one pick-and-drop by STYLE $\times$ LAYOUT.

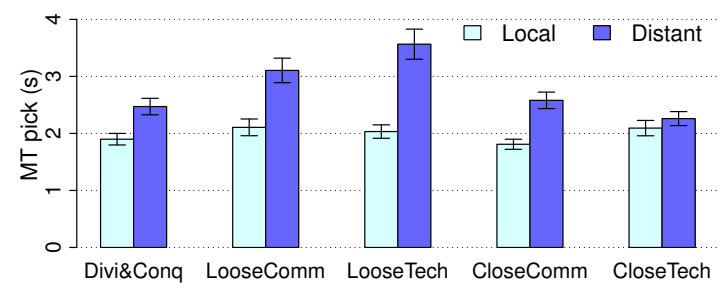

Figure 5. Mean time between a drop (or trial start) and the next pick by STYLE $\times$ LAYOUT.

each participant performing 8 pick-and-drops on average in parallel with her partner.

On the other hand, we found no significant difference between Divide\&Conquer, LooseComm and LooseTech ( $p$ 's = 1.0) even though $M T_{P D}$ for LooseTech is $7 \%$ faster than for Divide\&Conquer. This suggests that the benefit of helping each other is offset by the cost of collaboration, whether or not drop-for-partner is available. This is likely due to multitasking one's task with the partner's requests for help.

\section{Multitasking Cost}

To further understand the cost of collaboration, we analyze the time between subsequent pick-and-drop actions, i.e., from a drop to the next pick (Figure 5). We observed that in general the participants immediately picked a nearby red disc after dropping the previous one, if they were not distracted or interrupted by the partner. Therefore this measure reflects the level of distraction or multitasking cost. As we can see, this measure is almost constant for Local layouts.

For Distant layouts however, there are large significant differences between loose collaboration (LooseComm and LooseTech) and the other styles ( $p$ 's $<0.001$ for LooseTech and ( $p$ 's $<0.03$ for LooseComm). This suggests that helping each other while working in parallel has a cost, especially for LooseTech where participants helped each other a lot, and this offsets the small advantage noticed for LooseTech for pick-and-drop. This is consistent with the experimenters' observation that a participant would sometimes get interrupted and stop her own task to help her partner. Participants' subjective assessment also supports this finding (see below).

To confirm this finding, we measured the operational parallelization of the pick-and-drops in the parallel styles: the percentage of pick-and-drop overlap is the percentage of time of the overall task when both participants have picked a disc. For Distant layouts, this percentage is significantly lower for LooseTech (26\% of TCT) than for Divide\& Conquer $(p<0.001$, $41 \%$ of TCT) and LooseComm ( $p=0.008,35 \%$ of TCT). Referring back to the amount of collaboration (Fig. 2), this suggests that more collaboration leads to less parallelization. 

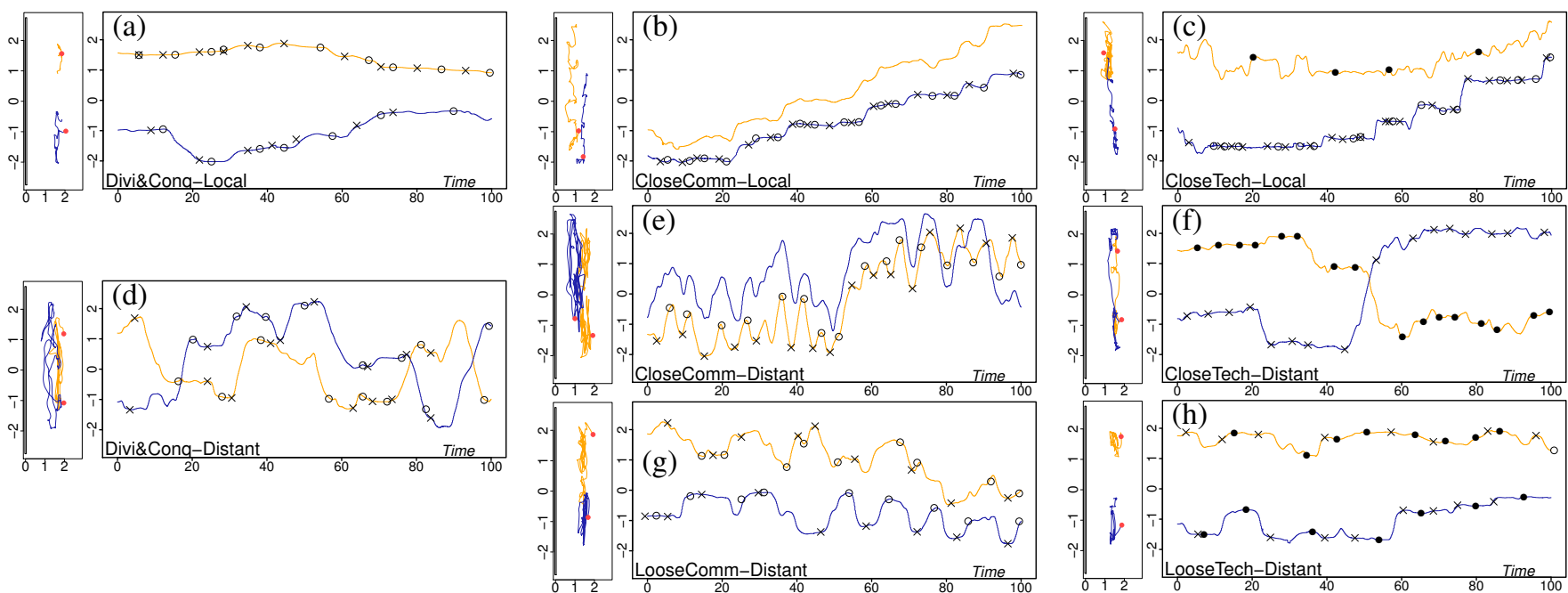

Figure 7. Navigation paths of eight pairs in different STYLE $\times$ LAYOUT conditions. LooseComm-Local and LooseTech-Local are omitted as they are very similar to Divide\&Conquer-Local (top-left graph). Each graph shows: on the left, the movement of both participants in a bird's eye view of the wall room with the wall on the left (unit is meter); on the right, the same paths stretched over a normalized timeline (x-axis) to help understand the pair's navigation patterns. In addition, picks $(\times)$, drops $(\circ)$ and drop-for-partner $(\bullet)$ actions are plotted on the paths.

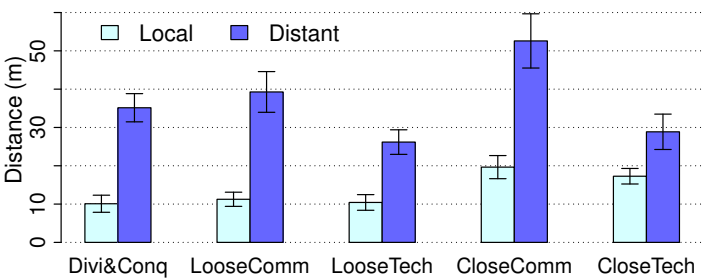

Figure 6. Mean total distance traveled by each pair, by STYLE $\times$ LAYOUT.

\section{Physical Navigation}

We first analyze the distance traveled by each pair (Figure 6). With Distant layouts, it is significantly shorter for LooseTech than for Divide\&Conquer ( $p=0.0013,26 \%$ shorter) and Loose Comm ( $p=0.0003,33 \%$ shorter). The same trend holds for CloseTech versus Divide\&Conquer $(p=0.1,17 \%$ shorter but not significant) and versus LooseComm ( $p=0.0076,26 \%$ shorter). This shows that the drop-for-partner feature can reduce travel distances when data is scattered.

To understand the coupling patterns for each condition we plot the movements of the participants during a trial in each condition (see Figure 7 for typical examples). By analyzing the participants' trajectories together with the amount of collaboration between partners (Figure 2), we find evidence of different "territoriality" and trajectory patterns according to the styles and layouts.

\section{Side-by-side vs. At-a-distance Collaboration}

We notice a trajectory pattern where one partner follows the other in all close collaboration conditions except CloseTech with Distant layouts. In these conditions, one participant typically follows the other and the task is solved together (e.g., Figure 7-e). This corresponds to a high level of effective collaboration.

Interestingly, CloseTech-Distant does not exhibit this "following" pattern, although the rate of effective collaboration is high $(74 \%)$. This means that the shared interaction tech- nique enables tight collaboration at a distance when the data to be manipulated is scattered (Figure 7-f).

\section{Territoriality}

As expected given the results on effective collaboration, we find very similar trajectory patterns for Divide \&Conquer and loose collaboration styles for Local layouts (Figure 7-a). Participants split the wall-sized display in two and perform the tasks independently.

With Divide\&Conquer and Distant layouts, trajectories cross the entire space, showing that participants work independently of each other (Figure 7-d). No territoriality appears in this condition.

With LooseComm and Distant layouts, partners tend to work on different areas of the wall-sized display. In fact, both LooseTech and CloseTech with Distant layouts also lead to user territories because the partners could share the search at a distance and use drop-for-partner (see Figure 2). Moreover, in CloseTech collaboration, participants often swapped the part of the wall they were working on so that the "picker" was closer to the misclassified discs (Figure 7-f).

\section{Combination of Power}

The drop-for-partner shared interaction technique makes it easier for partners to share both mental and physical resources at a distance. It augments the capabilities of single users by supporting a new form of collaboration.

Interesting evidence can be seen in Figure 8, which plots all self-dropping positions (in black) and drop-for-partner positions (in red) relative to the head position (graph center) of the person who picked the discs, for Distant layouts. The concentration of drops is the highest for Divide \& Conquer because, without help from their partner, participants can only read the labels in a small area in front of them. When communicating with their partner, some drop positions are further away from the participants for LooseComm and CloseComm 

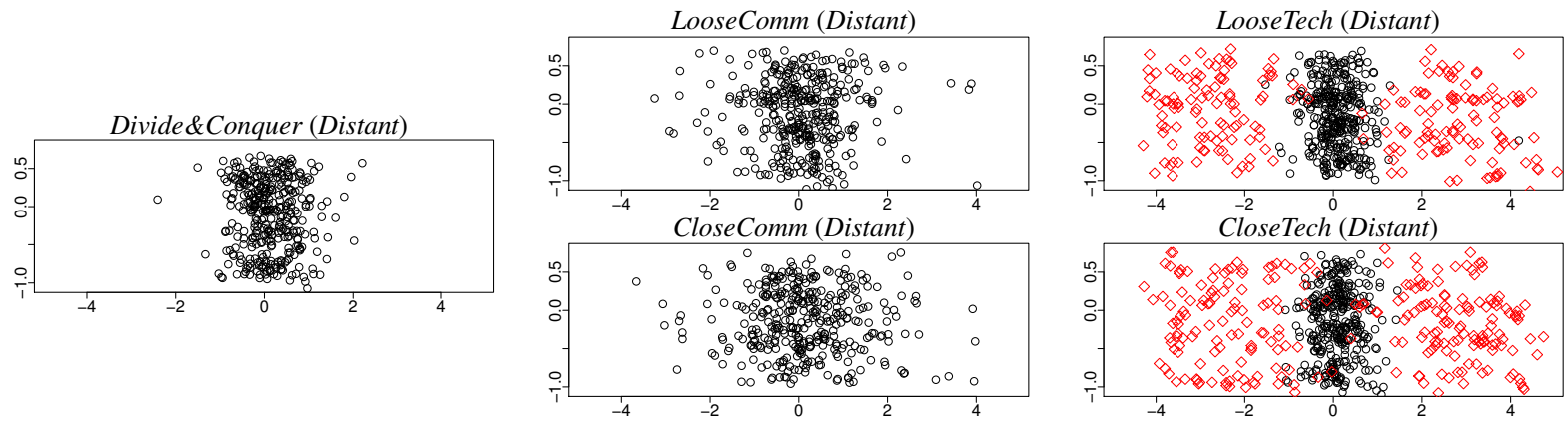

Figure 8. Positions of the drops relatively to the head of the picker (projected at the graph center $(0,0)$ ) for all participants, by STYLE for Distant layouts. The black circles $(\circ)$ show the drops performed using standard pick-and-drop. The red diamonds $(\diamond)$ show the drops performed using drop-for-partner.

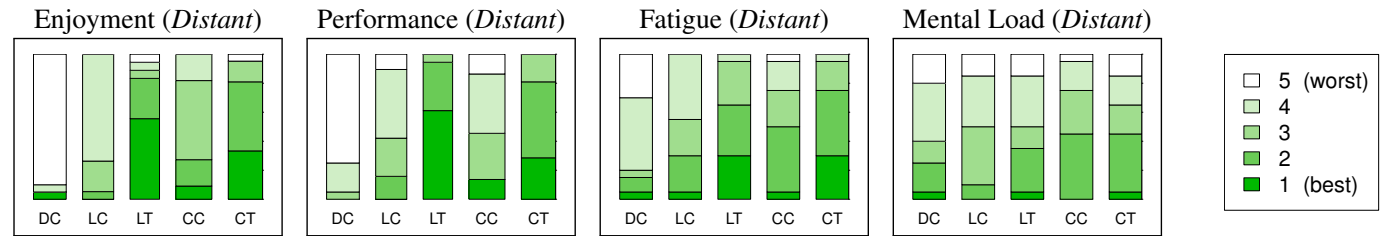

Figure 9. Participant ratings by STYLE for Distant layouts for Enjoyment and Perceived Performance (ranked from 1st to 5th) and for Fatigue and Mental Load on a five-point Likert scale (1 is best, 5 is worst). DC: Divide\&Conquer, LC: LooseComm, LT: LooseTech, CC:CloseComm, CT: CloseTech.

because they can drop into a container shown by their partner without being able to read the label. Furthermore, the participants' reach extends to the entire display with the support of drop-for-partner (LooseTech and CloseTech).

This shows that the provided shared interaction technique empowers the users' ability to perform the task, by letting them share both their knowledge and their physical ability. It encourages close collaboration and enables new forms of division of labor.

\section{Subjective Assessment}

At the end of the experiment we asked the participants to rank their perceived efficiency and enjoyment and to rate their physical and mental fatigue.

For Local layouts, Divide\&Conquer was perceived faster than LooseTech $(p=0.023)$ and CloseComm $(p=0.009)$, and marginally faster than CloseTech $(p=0.080)$. Other comparisons do not show significant differences.

Figure 9 shows the results for Distant layouts. The surprising result is that for Distant layouts, Divide\&Conquer was perceived as the slowest $(p$ 's $<0.005)$. Fifteen participants ranked Divide\&Conquer as the least efficient and four as the second least efficient while it is in fact the fastest style (Figure 3). The ranking of both perceived efficiency and enjoyment for LooseTech and CloseTech are significantly higher than for the other styles $(p$ 's $<0.01)$. It seems that perceived efficiency was largely influenced by how effective the collaboration was: "I was the most satisfied by the conditions where I felt we were working efficiently as a team and making the most progress quickly"; "I would say that the ones I enjoyed the most were the one where we collaborated the most".

Probably for the same reason, the perceived efficiency of CloseComm is higher than Divide \& Conquer while it is in fact the slowest. Indeed, enjoyment for CloseComm is ranked significantly higher than Divide\&Conquer and LooseComm ( $p$ 's
$<0.01$ ). One participant said, "CloseComm requires less synchronization and we can anticipate actions of our partner by focusing on his behavior (pointer and spatial location)".

In terms of physical fatigue, Divide\&Conquer was the most tiring style. ( $p<0.001$ compared to LooseTech and CloseTech, $p=0.013$ compared to CloseComm and $p=0.046$ compared to LooseComm). LooseTech was less tiring than LooseComm $(p=0.019)$ and CloseTech was marginally less tiring than CloseComm $(p=0.09)$. This corresponds to the measured travel distances, which are shorter for the styles providing the drop-for-partner feature.

Eighteen out of 20 participants confirmed that they were multi-tasking in LooseComm and LooseTech conditions: they were searching for the containers for themselves and their partner concurrently. Six of them thought it did not work well. There is no significant difference between styles for the ratings of mental load by LAYOUT ( $p$ 's $>0.2$ ) but mental load for Distant layouts is significantly higher than for Local layouts $(p<0.001)$. Indeed, 17 participants tried to remember the Distant layouts using spatial memory, and 2 gave up due to the heavy mental demand. Moreover, 11 participants mentioned that multi-tasking is mentally demanding, while a few others complained about the effort of verbal coordination and memorization of the layout.

\section{Summary}

With respect to our original research questions, the results provide evidence of the following interesting phenomena. First, participants value collaboration even though it incurs a cost. Indeed, while Divide\&Conquer was faster than the other styles, it was perceived as the least efficient and was the least preferred by the participants. Also, the navigation paths exhibit distinct patterns according to the collaboration style. Second, shared interaction increases collaboration, reduces physical navigation, improves operation efficiency and provides a more enjoyable experience. Third, distant layouts increase the value of collaboration and shared interaction. 


\section{DISCUSSION AND IMPLICATIONS FOR DESIGN}

The results of this study provide valuable input to improve collaboration and interaction efficiency and to create shared interaction techniques.

\section{Collaboration and Interaction Efficiency}

The goal of comparing the interaction efficiency of different collaboration styles is not to tell users which collaboration strategy is "best", but to gain a better understanding of the characteristics of these collaboration styles. Indeed, we see distinctive behavior patterns in each collaborative style that would be very difficult or impossible to observe in a realworld setting. Moreover, users tend to value efficient interactions because they do not want interaction with the system to get in the way of their collaborative task. More efficient interactions may encourage users to explore more possibilities and allocate more resources to problem solving and discussion.

In our observations of real users performing collaborative tasks, their main motivation is often to combine their knowledge and spark ideas through discussion. Hence, although our study suggests that communication may hinder interaction efficiency, this does not mean that communication is not beneficial to the users. For instance, in our experiment, less collaboration was perceived as less efficient and less enjoyable, leading to a lower level of engagement. Thus, interaction designers should consider the relative weights of efficiency, fatigue and fun when designing for a real-world task, according to its nature, e.g. creative vs. time-critical task.

Our analysis also shows that increasing collaboration in loosely collaborative conditions adds a cognitive cost because of multi-tasking and disruption by the partners. A design implication is to provide interaction techniques that support collaboration while minimizing the cost of disruption.

\section{Shared Interaction}

We can see several benefits of providing shared interaction techniques to support collaboration. First, such techniques can improve collaboration. For example, our participants collaborated much more when they could use the drop-forpartner technique to move objects across the wall.

Shared interaction techniques can also improve efficiency: drop-for-partner improved the efficiency of individual pickand-drops and reduced travel distances and physical fatigue. This is especially beneficial when tight collaboration is needed at a distance. Existing collaboration techniques are rarely designed for this purpose nor used in this context. This opens an interesting design space to explore new techniques.

Drop-for-partner also altered the trajectory patterns of participants. The existing literature on large displays often states that users collaborate more when they are physically close. Our work demonstrates that shared interaction techniques can support tightly-coupled collaboration at a distance, thus allowing different forms of division of labor in certain contexts.

Drop-for-partner is a specific instance of a shared interaction technique, designed for pairs to move content around a wall-sized display. Other techniques should be developed that match the characteristics of the collaborative task. Designers should observe the communication patterns among users and design shared interaction techniques that support them.

\section{CONCLUSION}

In this work we operationalized and compared five collaboration styles: a baseline condition and four styles resulting from crossing two dimensions - collaborative coupling and shared interaction support. We conducted an experiment with an abstract data manipulation task that allowed us to focus on the interaction level of collaboration and compare the collaboration styles using quantitative measures. This methodology complements observational studies that often deal with occurrences of different collaboration styles within a single task and do not attempt to control the various conditions. While this one experiment necessarily picked a particular task and interaction technique, the methodology can be applied with other factors and interaction techniques in different contexts.

Our results show that providing a shared interaction technique can improve collaboration by letting co-workers collaborate more tightly even when not in close proximity. Shared interaction also increases efficiency, reduces physical travel and improves subjective experience. Furthermore, the detailed analyses of individual interactions help quantify part of the collaboration cost incurred by multitasking and interruption. These findings can inform the design of shared interaction techniques to support collaboration on wall-sized displays.

\section{Future Work}

This study has focused on the interaction level of a collaborative task, justifying our choice of a task with minimal cognitive demand. However, tasks that involve intellectual communication and negotiation such as sense-making tasks or generative tasks such as brainstorming could be studied with a similar approach in order to determine if specific shared interaction techniques can improve collaborative work.

The drop-for-partner feature that we introduced is a simple example of a shared interaction technique. More advanced techniques such as multi-selection, grouping or editing should be tested, as they may lead to different trade-offs and new collaboration styles. Future work should explore the design space of shared interaction techniques for wall-sized displays, and evaluate them with different tasks and collaboration scenarios such as users with different roles or levels of expertise. Finally we only considered pairs of colocated users. Future work should also study larger groups, with more complex structures, in both colocated and remote settings.

An interesting finding of this work is the mismatch between actual and perceived efficiency of some collaboration styles. This raises challenging questions about the metrics to be used to assess collaborative activities. Should enjoyment be used instead of task completion time? Or can we reconcile the social qualities of collaborative interaction with raw efficiency?

\section{ACKNOWLEDGEMENTS}

This research was partially supported by Labex DigiCosme (Idex Paris-Saclay ANR-11-IDEX-0003-02) and Digiscope (EquipEx ANR-10-EQPX-26-01), both operated by ANR as part of the program "Investissements d'Avenir". 


\section{REFERENCES}

1. Christopher Andrews, Alex Endert, and Chris North. 2010. Space to think: Large high-resolution displays for sensemaking. In Proceedings of the SIGCHI Conference on Human Factors in Computing Systems (CHI '10). ACM, 55-64. DOI : 10.1145/1753326.1753336

2. Robert Ball, Chris North, and Doug A. Bowman. 2007. Move to improve: promoting physical navigation to increase user performance with large displays. In Proceedings of the SIGCHI Conference on Human Factors in Computing Systems (CHI '07). ACM, 191-200. DOI : 10.1145/1240624.1240656

3. Lauren Bradel, Alex Endert, Kristen Koch, Christopher Andrews, and Chris North. 2013. Large high resolution displays for co-located collaborative sensemaking: Display usage and territoriality. Int. J. Hum.-Comput. Stud. 71, 11, 1078-1088. DOI: 10.1016/j.j.jhcs.2013.07.004

4. Paul Dourish and Victoria Bellotti. 1992. Awareness and coordination in shared workspaces. In Proceedings of the 1992 ACM Conference on Computer-supported Cooperative Work (CSCW' '92). ACM, 107-114. DOI: $10.1145 / 143457.143468$

5. Michael Fitze. 2006. Discourse and participation in ESL face-to-face and written electronic conferences. Language Learning \& Technology 10, 1, 67-86. http://www.editlib.org/p/74430/

6. Carl Gutwin and Saul Greenberg. 2002. A descriptive framework of workspace awareness for real-time groupware. Comput. Supported Coop. Work 11, 3, 411-446. DOI : 10.1023/A:1021271517844

7. Kirstie Hawkey, Melanie Kellar, Derek Reilly, Tara Whalen, and Kori M. Inkpen. 2005. The proximity factor: Impact of distance on co-located collaboration. In Proceedings of the 2005 International ACM SIGGROUP Conference on Supporting Group Work (GROUP '05). ACM, 31-40. DOI: 10.1145/1099203.1099209

8. Juan David Hincapié-Ramos, Xiang Guo, Paymahn Moghadasian, and Pourang Irani. 2014. Consumed endurance: A metric to quantify arm fatigue of mid-air interactions. In Proceedings of the SIGCHI Conference on Human Factors in Computing Systems (CHI '14). ACM, 1063-1072. DOI: 10.1145/2556288.2557130

9. Eva Hornecker, Paul Marshall, Nick Sheep Dalton, and Yvonne Rogers. 2008. Collaboration and interference: Awareness with mice or touch input. In Proceedings of the 2008 ACM Conference on Computer Supported Cooperative Work (CSCW'08). ACM, 167-176. DoI: 10.1145/1460563.1460589

10. Petra Isenberg, Danyel Fisher, Sharoda A. Paul, Meredith Ringel Morris, Kori Inkpen, and Mary Czerwinski. 2012. Co-located collaborative visual analytics around a tabletop display. IEEE Trans. Visual. Comput. Graphics 18, 5, 689-702. DOI:

10.1109/TVCG.2011.287
11. Mikkel R. Jakobsen and Kasper Hornbaek. 2014. Up close and personal: Collaborative work on a high-resolution multitouch wall display. ACM Trans. Comput.-Hum. Interact. 21, 2, Article 11, 34 pages. DOI : 10.1145/2576099

12. Mikkel R. Jakobsen and Kasper Hornbæk. 2015. Is moving improving?: Some effects of locomotion in wall-display interaction. In Proceedings of the 33rd Annual ACM Conference on Human Factors in Computing Systems (CHI '15). ACM, 4169-4178. DOI: 10.1145/2702123.2702312

13. Can Liu, Olivier Chapuis, Michel Beaudouin-Lafon, Eric Lecolinet, and Wendy Mackay. 2014. Effects of display size and navigation type on a classification task. In Proceedings of the SIGCHI Conference on Human Factors in Computing Systems (CHI '14). ACM, 4147-4156. DOI : $10.1145 / 2556288.2557020$

14. Meredith Ringel Morris, Anthony Cassanego, Andreas Paepcke, Terry Winograd, Anne Marie Piper, and Anqi Huang. 2006a. Mediating group dynamics through tabletop interface design. IEEE Comput. Graph. Appl. 26, 5, 65-73. DOI : 10.1109/MCG.2006.114

15. Meredith Ringel Morris, Anqi Huang, Andreas Paepcke, and Terry Winograd. 2006b. Cooperative gestures: Multi-user gestural interactions for co-located groupware. In Proceedings of the SIGCHI Conference on Human Factors in Computing Systems (CHI '06). ACM, 1201-1210. DOI : 10.1145/1124772.1124952

16. Miguel A. Nacenta, David Pinelle, Dane Stuckel, and Carl Gutwin. 2007. The effects of interaction technique on coordination in tabletop groupware. In Proceedings of Graphics Interface 2007 (GI '07). ACM, 191-198. DOI : $10.1145 / 1268517.1268550$

17. Tao Ni, Doug A. Bowman, and Jian Chen. 2006. Increased display size and resolution improve task performance in information-rich virtual environments. In Proceedings of Graphics Interface (GI '06). CIPS, 139-146.

http : //dl . acm.org/citation. cfm?id=1143079. 1143102

18. Emmanuel Pietriga, Stéphane Huot, Mathieu Nancel, and Romain Primet. 2011. Rapid development of user interfaces on cluster-driven wall displays with jBricks. In Proceedings of the 3rd ACM SIGCHI symposium on Engineering interactive computing systems (EICS '11). ACM, 185-190. DOI : 10.1145/1996461.1996518

19. David Pinelle, Carl Gutwin, and Saul Greenberg. 2003. Task analysis for groupware usability evaluation: Modeling shared-workspace tasks with the mechanics of collaboration. ACM Trans. Comput.-Hum. Interact. 10, 4, 281-311. DOI : 10.1145/966930.966932

20. Gonzalo Ramos, Kenneth Hinckley, Andy Wilson, and Raman Sarin. 2009. Synchronous gestures in multi-display environments. Hum.-Comput. Interact. 24, 1-2, 117-169. DOI: 10.1080/07370020902739288 
21. Khairi Reda, Andrew E. Johnson, Michael E. Papka, and Jason Leigh. 2015. Effects of display size and resolution on user behavior and insight acquisition in visual exploration. In Proceedings of the 33rd Annual ACM Conference on Human Factors in Computing Systems (CHI'15). ACM, 2759-2768. DOI : 10.1145/2702123.2702406

22. Roy A. Ruddle, Rhys G. Thomas, Rebecca S. Randell, Phil Quirke, and Darren Treanor. 2015. Performance and interaction behaviour during visual search on large, high-resolution displays. Information Visualization 14, 2, 137-147. DOI : 10.1177/1473871613500978

23. Stacey D. Scott, M. Sheelagh T. Carpendale, and Kori M. Inkpen. 2004. Territoriality in collaborative tabletop workspaces. In Proceedings of the 2004 ACM Conference on Computer Supported Cooperative Work (CSCW '04). ACM, 294-303. DOI: 10.1145/1031607.1031655

24. Desney S. Tan, Darren Gergle, Regan Mandryk, Kori Inkpen, Melanie Kellar, Kirstie Hawkey, and Mary Czerwinski. 2008. Using job-shop scheduling tasks for evaluating collocated collaboration. Pers. Ubiquit. Comput. 12, 3, 255-267. DOI : 10.1007/s00779-007-0154-3

25. Anthony Tang, Melanie Tory, Barry Po, Petra Neumann, and Sheelagh Carpendale. 2006. Collaborative coupling over tabletop displays. In Proceedings of the SIGCHI Conference on Human Factors in Computing Systems (CHI 'O6). ACM, 1181-1190. DOI : 10.1145/1124772.1124950

26. Edward Tse, Jonathan Histon, Stacey D. Scott, and Saul Greenberg. 2004. Avoiding interference: How people use spatial separation and partitioning in SDG workspaces. In Proceedings of the 2004 ACM Conference on Computer Supported Cooperative Work (CSCW '04). ACM, 252-261. DOI : 10.1145/1031607.1031647

27. James R. Wallace, Stacey D. Scott, and Carolyn G. MacGregor. 2013. Collaborative sensemaking on a digital tabletop and personal tablets: Prioritization, comparisons, and tableaux. In Proceedings of the SIGCHI Conference on Human Factors in Computing Systems (CHI'13). ACM, 3345-3354. DOI: $10.1145 / 2470654.2466458$

28. Nicola Yuill and Yvonne Rogers. 2012. Mechanisms for collaboration: A design and evaluation framework for multi-user interfaces. ACM Trans. Comput.-Hum.

Interact. 19, 1, Article 1, 25 pages. DOI: 10.1145/2147783.2147784 